Usage, Usability, and Utility of 3D City Models, 02007 (2012)

DOI: $10.1051 / 3 \mathrm{u} 3 \mathrm{~d} / 201202007$

(c) Owned by the authors, published by EDP Sciences, 2012

\title{
Developing an ontology of space: Application to 3D city modeling
}

\author{
R. Billen ${ }^{1, a}$, C. Zaki ${ }^{2, b}$, M. Servières ${ }^{2, b}$, G. Moreau ${ }^{2, b}$ and P. Hallot ${ }^{1, a}$ \\ ${ }^{1}$ Université de Liège, Unité de Géomatique, Belgique \\ ${ }^{2}$ LUNAM Université, École Centrale Nantes, CERMA UMR CNRS 1563, France
}

\begin{abstract}
Although information modeling techniques and ontologies developments have been intensively used and studied, we believe that clear and logical links between these different concepts are missing. It is even more crucial in 3D city modeling context where standardization processes are less developed. In this paper, we first give an unambiguous view of the interrelations and dependencies between models and ontologies based on a top-level characterization of data, knowledge and information. We then propose an ontology of space aiming at strengthening basis of 3D City models, with a specific application to some objects of the CityGML 3D geo-information standard.
\end{abstract}

\section{INTRODUCTION}

Existing technologies provide means to organize and structure data in order to facilitate their storage and handling. "Modeling" is part of the procedure that ensures the transition from the real-world to the computing world. Modeling data may require several levels of abstraction. Traditionally, the approach of modeling, as recognized by the ANSI (American National Standards Institute), includes a step of perception of the real world (including the analysis of user problems and needs), followed by a sequence of three modeling steps: conceptual, logical and physical. This approach is designer / enduser-oriented: it implies structures, concepts and meta-models existence as well as prior formalization, non visible for the end-user. Implicitly or less often explicitly, an ontological level stands on the top of this approach, which is usually non-accessible for the end-user. Geographic data are specific in the sense that they refer to characteristics of different scales: attribute, spatial and temporal. Their models and their representations have been at the origin of a vast literature over the past two decades. However this diversity and multiplicity of actors and researches in this area have resulted in syntactic as well as semantic heterogeneities of data and reduced their interoperability.

Syntactic interoperability addresses the technical level (eg, communications protocol, representation models and formats, etc.), while semantic interoperability is concerned with domain knowledge required for computer services to "understand" the intentions and capabilities of each service. To address the problems of interoperability, standards for geographic information have been developed by standardization organisms: Open Geospatial Consortium (OGC) or ISO/TC211 (ISO Technical Committee for Geographic Information and Geomatics). The use of these standards has gradually removed the difficulties resulting from data structure incompatibilities and syntax but does not allow solving all the problems stemming from the semantic heterogeneity.

One of the most explored directions to address this last problem is the use of ontologies. Ontology is a formal technique for representing knowledge; it defines a concise conceptual basis for expert knowledge sharing and data interoperability, and offers users the ability to define and structure the

\footnotetext{
a e-mail: $\{$ p.hallot, rbillen\}@ulg.ac.be

b e-mail: \{chamseddine.zaki, myriam.servieres, guillaume.moreau\}@cerma.archi.fr
}

This is an Open Access article distributed under the terms of the Creative Commons Attribution License 2.0, which permits unrestricted use, distribution, and reproduction in any medium, provided the original work is properly cited. 
semantics of terms and concepts used in a given field. Knowledge explicit definition using ontologies is commonly used as a mechanism to understand and resolve semantic heterogeneities occurring when combining multiple systems. Ontology has become a key area of research in geographical information science, but developments rarely cross laboratories frontiers and most of these works remain largely on paper or in prototype stage.

We have notices that the relationships between ontology, standard, meta-model, conceptual model, logical model, physical model, etc. are not always clearly understood; it is at least the case in the geo-information domain. However, to move logically from one type to another and ensure hierarchical consistency between them, it is essential to clearly define the scope and significance of each type. We emphasize that from our point of view (as we will detail later in this article) all of these types are only techniques used to represent knowledge and organize data at different levels of abstraction. Together, they build a logical sequence to identify and explain the formal description and organization of data at different levels of detail.

Clarifying these aspects is especially important in the context of $3 \mathrm{D}$ city modeling. Indeed, the standardization processes are less developed compared to 2D geo-information; CityGML is one of the only developments in this area and is supported by the OGC. The potential interoperability issues are much bigger than in 2D and further formalization effort is needed. This lack of standardization can be explained in two ways: part of the geo-information community consider 3D as a simple extension of 2D objects; but there is a much higher diversity of communities working with 3D city models, which makes the integration issue even more dramatic.

Consequently, we have decided to initiate the development of an ontology aiming at representing (3D) spatial knowledge inferred from our experience in the field of data acquisition. We establish links between this ontology and CityGML considering that a data model needs to be built on explicit ontological concepts to be stable, solid and consistent, avoiding the pitfalls of heterogeneity.

In this paper, we start with a global vision of the data and knowledge characterization and representation (section 2). Then, we recall important definitions allowing a better articulation of concepts such as models and ontologies (section 3). Section 4 presents another level of our global approach linking the different types of characterization and representation of knowledge and data defined in section 3. In Section 5, we develop different levels of the proposed ontology of space. Then, in section 6, we apply this ontology in the 3D city modeling context, with a specific focus on some objects of the CityGML. Finally we conclude in section 7.

\section{DATA AND KNOWLEDGE MODELING}

The purpose of characterization is to represent the real world and a selection of expert knowledge in a computerized form. In other words, it is a framed representation of the real world according to application needs and requirements of specific case studies. It is also a representation of experiences in a certain field to organize and fit its use.

This characterization involves several stages of knowledge representation and data modeling, according to various levels of abstraction. Their benefits are that they allow representing and matching the case of applications in a manner that is both easy to understand by the user and usable by the computer.

Our characterization approach is multi-level and hierarchical. The main rule is that the lower levels are more specific than higher levels but they enforce their constraints. The assembly can be seen as a pyramidal structure, allowing the resolution of specific problems at lower levels with reference to constraints formally imposed by the higher levels. The top level, called level- 0 gathers data, information, knowledge, phenomena and thought concepts. These concepts and their interactions have been heavily studied [1,2] and are essential in various domains such as knowledge management, economy, etc [3]. Obviously, level-0 (Figure 1) is an incomplete vision of reality that has been chosen as a starting point, but the latter could be changed for the sake of generality. 


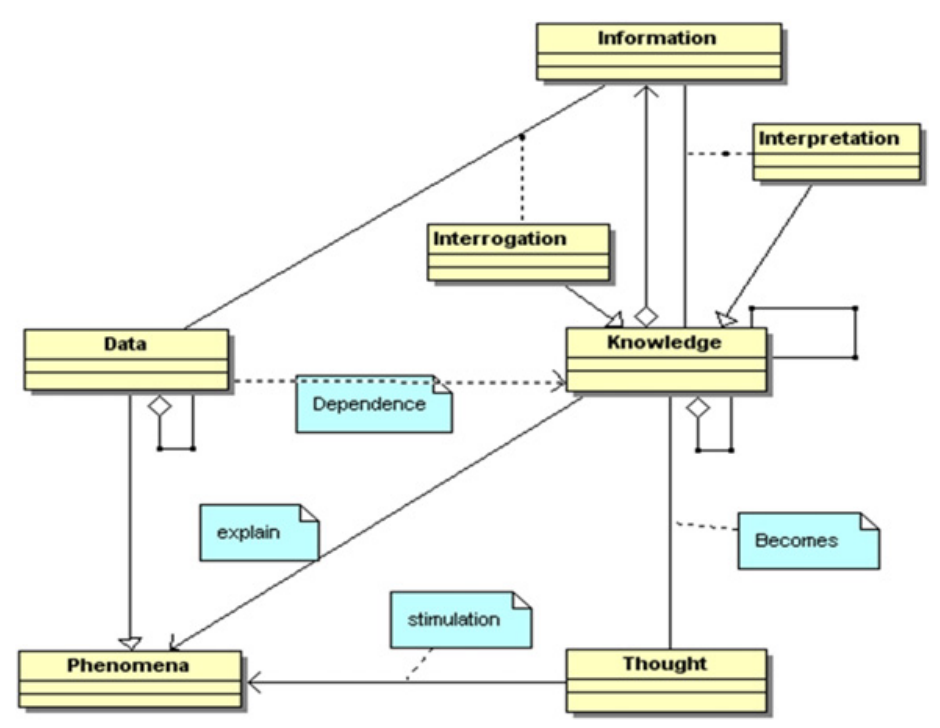

Figure 1. Level-0 of the approach. This is indeed an ontology represented by a conceptual schema.

The phenomena are the most abstract concept in this scheme. There is a relationship with thoughts (ideas). The observation (happening) of phenomena stimulates thoughts. (The oriented relation "stimulation" between phenomena and thought means that ideas can be "stimulated by" a phenomena).

Thoughts are a source of knowledge. Indeed, an association "Becomes" exists between thoughts and knowledge. It results from a repetition, accumulation and / or intuition.

Knowledge has a reflexive relationship that allows, through interpretation and deduction, to produce new knowledge from existing knowledge. Knowledge is composed of sub knowledge and helps giving meaning (explaining) phenomena.

Data are phenomena explained by knowledge and consequently become measurable. The data may contain data (data composed of sub data) and product information after questioning. The information is the result of the queried data (through interrogation) or of the interpretation of knowledge and are a component of knowledge. Data and knowledge are sources of information. Querying and interpretation are specific types of knowledge.

In our characterization approach, there are complementarities and interactivities between reality and the computerization of reality. Computerization is part of the real world and the real world is a toplevel part of its computerization. The difference is that the computer world is an evolving vision but temporally bounded and closed by technological advancement, while the real world is open in terms of its interpretation by man.

\section{MODELS AND ONTOLOGIES}

In our work of information characterization we differentiate data modeling (part of characterization oriented end-designer) from knowledge representation (part of characterization oriented domains experts).

\subsection{Data modeling}

Modeling data includes a step of observation of the real world that is focused on the expression of users' problems and needs, followed by three main levels of data modeling: the conceptual level, the logical level and the physical level. 
- Conceptual level: The conceptual level is an easy way to understand data organization. It is an intermediate level between the observed reality (specific data) and the computer. The conceptual model is a formal framework to represent the content of information whereas the conceptual schema is a description (mapping) of the database. In other words, it represents the case study, designed by the designer according to a formal model on precise specifications, and facilitates the understanding and the usage of the application. Interested readers can refer to [4].

- Logical level: The logical level is an intermediate level between the conceptual level and the system. The logical schema is the result of the transformation of the conceptual schema into a data structure supported by the model (for example, tables for a relational data model and classes for an object data model).

- Physical level: The physical level is data storage level. The physical schema is a translation of the logical diagram according to the syntax of the chosen DBMS (DataBase Management System).

In most cases the transition from the conceptual level to the physical level (transformation of the conceptual scheme in a physical one) is transparent and automatic. The physical level is less expressive than the conceptual level, so the automatic transformation must take into account the integration of methods and techniques to reduce this semantic mismatch [5]. Regarding the geographical scope, contributions and research have been undertaken in this level to respond effectively to the demands of the geographic area [5-9].

\subsection{Knowledge Representation: Ontologies and meta-models}

This section aims at clarifying concepts of ontologies and meta-models. Ontologies are a formal mechanism for knowledge representation and their use cover a broad spectrum of areas. A given ontology can be represented in a formal or informal way depending on its objective [10]. If the goal is to provide communication between people, a description in natural language may be sufficient. By cons, if the role of an exchange format is assumed in an environment of interoperability among programs, it is necessary that it be formal as long as the communication is consistent and the automatic verification of this consistency is possible.

Depending on their use, [11] distinguishes five types of ontologies: generic, domain, application, representation and methods [12].

- Generic ontologies describe general concepts that are independent of an area or a particular problem. These include, for example, the concepts of time, space, and events.

- Domain ontologies specify a point of view on a particular area (geographic domain for example). The concepts of domain ontology are often defined as a specialization of the concepts of generic ontologies.

- Application ontologies describe the structure of the knowledge needed to achieve a particular task [11]. They are used for example to facilitate the process of acquiring knowledge of the application.

- The representation ontology specifies a description formalism that provides a representative structure and primitives to describe the concepts of domain ontologies and generic ontologies [12].

- Ontologies of Methods, tasks and problem solving describe the reasoning process independently of a domain and a given implementation. They specify entities under the resolution of a problem such as abduction, deduction or observation [13].

On the side of knowledge representation, there are also meta-models. A meta-model is therefore the formal definition of the elements, rules, constraints, etc. that govern the creation of a model. The model, as the meta-model, is made using formalism. However, the same formalism can be used to build the meta-model as is the case for the UML. In this situation, the used language or formalism allows to describe itself. 


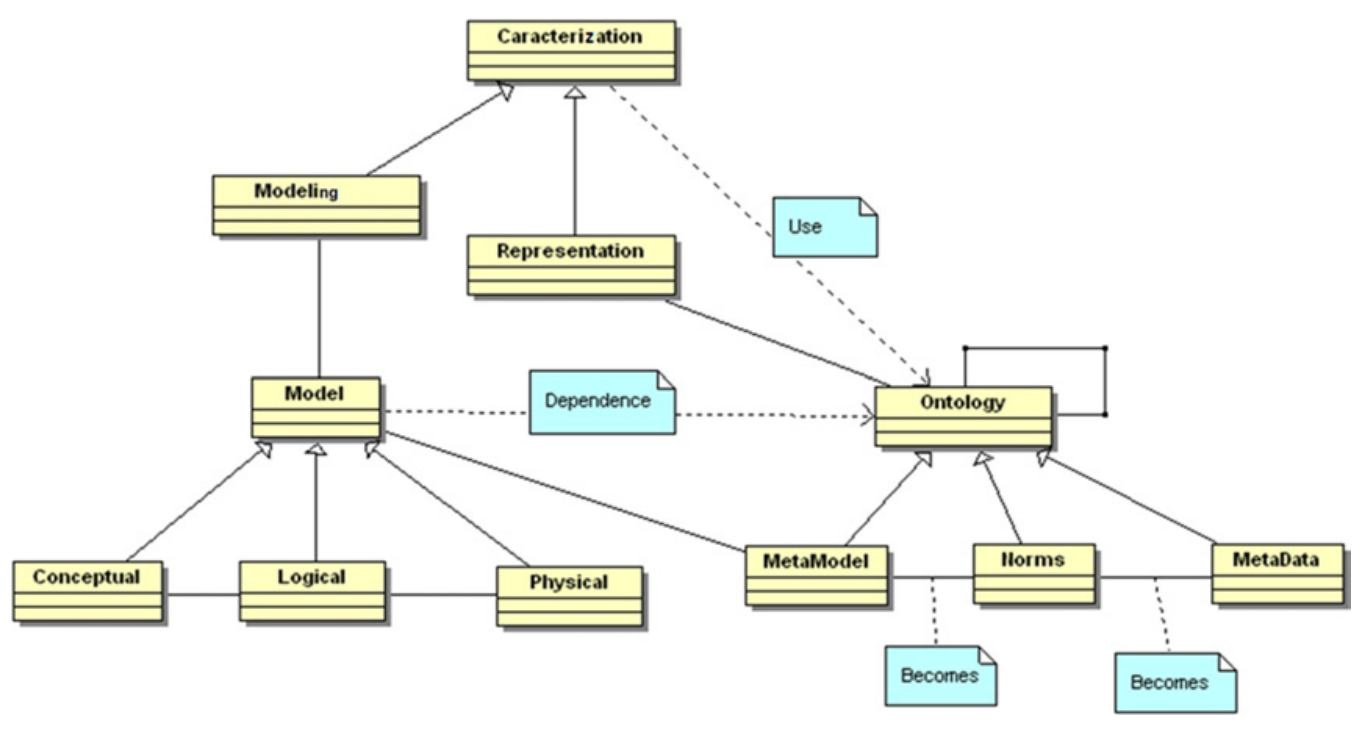

Figure 2. Projection of level-0 of characterization on the information technology domain.

From its definition, directly stems the utility of the meta-model, it is the strict framework that will dictate the use of a formalism and, therefore, resolve any ambiguities about the use of its concepts. It will directly allow understanding and explaining perfectly the models made from the formalism. Metamodeling has another interesting aspect. Since the meta-model itself is a model made from a formalism, this (meta-) model can be enriched in accordance with the formalism's concepts, concepts that are also described in the meta-model. This feature allows avoiding having to redefine basic concepts such as classes and associations and to borrow from a recognized and proven formalism as UML for example.

Thus, considering its definition, its utility and its role, the meta-model is for us a formalized knowledge representation technique that is specified for a particular area, and consequently a type of ontology (category compound).

Similarly to meta-models, Metadata are defined as data describing a given data. These are important elements in the knowledge, access, dissemination and proper use of geographic data. This information typically focuses on the nature of the data, its origin, availability, update frequency, quality, access or use. They are therefore essential to understanding and sharing of data by users over time.

\section{APPLICATION TO INFORMATION TECHNOLOGY}

The next diagram (Figure 2) represents the computer characterization of the real world. It outlines the various modes of knowledge representation, data modeling and relations between them as well as the different means of data manipulation and knowledge extraction. Indeed it is a projection from level-0 of characterization to the field of information technology. We did not specify in this figure the 5 subcategories of ontology, since at this level we are only interested by our general representation of the computer world.

The concept of "characterization" in this diagram corresponds to "information" from the real world (Figure 1). Modeling and representation are its two subtypes. Modeling provides data query and a (partial) framework for the creation of data models based on ontologies (there exists a dependency relationship between characterization and ontology). Modeling in this figure corresponds to information represented in Figure 1. Models are tools for creating patterns corresponding to particular application case studies. A model is created by a meta-model that describes it (association relationship between 


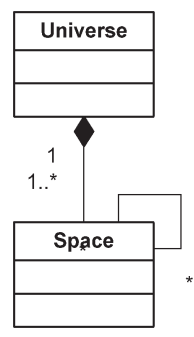

Figure 3. Proposed ontology of space - level 1.

model and meta-model in Figure 2). A model can be conceptual, logical or physical. The relationship between conceptual and logical is the fact that one or more logical patterns can be created from one conceptual (more expressive) schema. The association relationship between logical and physical models is due to the fact that a physical schema is created from a logical pattern. Representation provides the knowledge interpretation of ontologies (also based on specific ontologies; dependency relationship between characterization and ontology). Representation in this figure corresponds to knowledge in Figure 1. Meta-models, standards, metadata and taxonomies are subtypes of the ontology. Meta-model or meta-data ontology can be self-normalized. This knowledge is represented by the relationship "becomes" between "meta-model" and "standards" and the "becomes" relationship between "metadata" and "standards". Ontology has a reflexive relationship because of the fact that an ontology can be used, be based or have relationships with other ontologies.

\section{PROPOSED ONTOLOGY OF SPACE}

\subsection{Our approach}

Our approach was incepted thanks to discussions in the field of geo-information. Indeed, the various interpretations and definitions of "models" and "ontologies" caused a problem in our description of geographic information, especially in our works on 3D urban information. CityGML [14] was established as a new standard for 3D urban information, as a data exchange format but also considered as a data model for a wide community. We realized that this model, although widely well documented, was not based on any explicit ontology. Following our characterization approach, CityGML is a standard physical model that creates patterns of physical data, although formulated in a meta-model [15] that does not make reference to ontologies. We argue that to undertake a data modeling that is stable, solid and consistent, avoiding the pitfalls of heterogeneity, it is necessary to construct it conceptually and ontologically in an explicit way. In this spirit, we have initiated the development of a domain ontology (Ontology with different levels inspired from our experience in field of data acquisition) [16, 17] and tried to get in touch with the elements of CityGML. We use the UML meta-model to express our ontology, concept definitions are numbered as D.[level of the ontology].number, and relationships are numbered as R.[level of the ontology].number.

\subsection{Ontology levels}

\section{Level 1}

Our ontology starts with a concept of Space, n-dimensional (nD) partition of the Universe.

D.1.1 Universe is the top concept.

D.1.2 Space is a partition of Universe.

R.1.1 A Universe is composed of at least one Space / A Space composes only on Universe.

R.1.2 A Space can be in relation with other Spaces. 


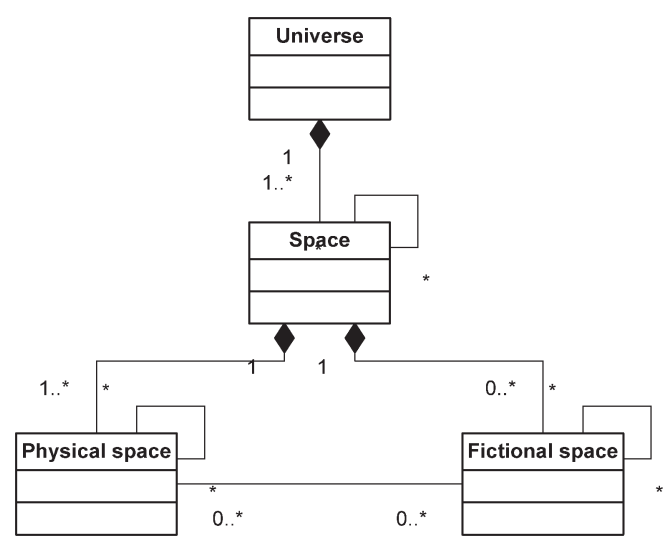

Figure 4. Proposed ontology of space - level 2.

\section{Level 2}

At this level, we consider two types of space: the physical space corresponding to a material "world" and the fictional space corresponding to an immaterial "world". Other types of spaces might exist but not being identified at this stage, they are not represented.

D.2.1 A Physical space is a $\mathrm{nD}$ portion of Space, composed of materials or vacuum.

D.2.2 A Fictional space is a portion of Space, composed of thoughts (immaterial).

R.2.1 A Space is composed of at least one Physical space / A Physical space composes only one Space.

R.2.2 A Space can be composed of one or several Fictional space / A Fictional space composes only one Space.

R.2.3 A Physical space can be in relation with other Physical spaces.

R.2.4 A Fictional space can be in relation with other Fictional spaces.

R.2.5 A Physical space can be in relation with Fictional spaces / A Fictional space can be in relation with Physical spaces.

\section{Level 3}

At this level we consider only the Physical space and its compositions. Fictional space and its compositions are not discussed in this paper. This level brings another simplification by considering only 3 dimensions. We consider that a Physical space can be externally connected with other Physical spaces, which means a connection without overlap. We consider that Physical spaces are limited by Boundaries. When two Physical spaces are externally connected, it means that part of their related Boundaries share exactly the same location. This latter assumption follows Brentano suggestions (cited in [18]).

D.3.1 A Physical sub-space is a portion of Physical space.

D.3.2 A Boundary is a 2D limit of a Physical space.

R.3.1 A Physical space can be composed of one or several Physical sub-space / A Physical sub-space composes only one Physical space.

R.3.2 Physical sub-space is a specialization of Physical space.

R.3.3 A Physical sub-space is always included in the Physical space it composes / A physical space covers its Physical sub-spaces components.

R.3.4 A Physical space has at least one Boundary / A Boundary belongs to one Physical space. 

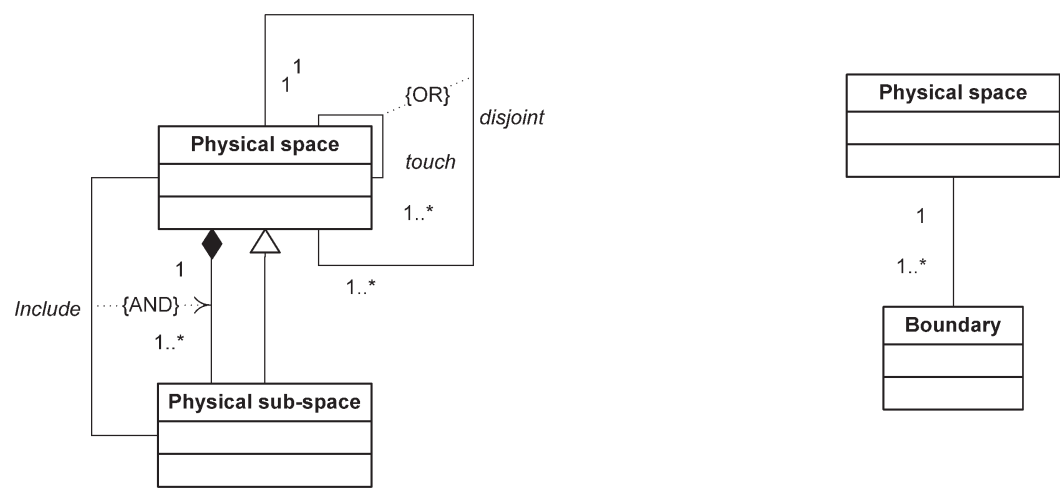

Figure 5. Proposed ontology of space - level 3.
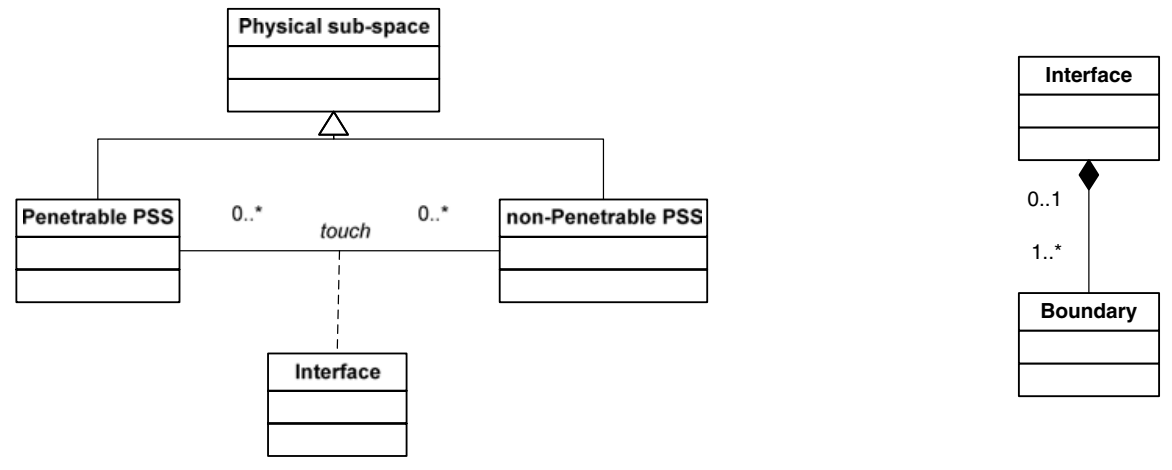

Figure 6. Proposed ontology of space - level 4.

R.3.5 Physical spaces which are not linked with a "part-of / whole" relationship, shares a touch or a disjoint relationships. A touch relation implies an exact collocation of Boundaries of two Physical spaces.

\section{Level 4}

At this level, we introduce the notion of agent, being in interaction with the Physical space. The underlying idea is that a Physical sub-space, depending of its material composition, can be or not penetrated by an agent. An agent can be an electromagnetic wave (XR, light, etc.), a physical object (a human), etc., something presenting a specific behavior (following a physical law, a cognitive behavior, etc.). Note that this notion of agent is not formally defined in the ontology; it has to be seen as a criterion for Physical sub-spaces specialization. For a given agent, Physical sub-spaces can be specialized into Penetrable physical sub-spaces (PPSS) and non-penetrable physical sub-spaces (nPPSS). A touch relationship between Physical spaces (R.3.5) can be specialized when involving PPSS and nPPSS; in such case, the Boundaries involved in the relationship compose an Interface. Interface is, for a given agent, the limit between the penetrable and the non-penetrable spaces.

D.3.1 Penetrable physical sub-space (PPSS) is a specialized Physical sub-space which is penetrable for a given agent.

D.3.2 non-Penetrable physical sub-space (nPPSS) is a specialized Physical sub-space which is non penetrable for a given agent. 
D.3.3 Interface is the limit between PPSS and nPPSS for a given agent.

R.3.1 Penetrable physical sub-space (PPSS) is a specialization of Physical sub-space.

R.3.2 non-Penetrable physical sub-space (nPPSS) is a specialization of Physical sub-space.

R.3.3 When there is a touch relation (cf. R.2.1) between a PPSS and a nPPSS, the corresponding contact zone is an Interface.

R.3.4 An Interface is composed of at least one Boundary / A Boundary may compose one Interface.

\section{Level 5}

At this level we introduce the World object that is a mind conceptualization. We adopt the well-known categorization proposed by [18] into bona fide objects and fiat objects. This categorization is based on object's boundaries which are themselves divided in bona fide and fiat boundaries. In brief, a bona fide boundary corresponds to a "real" physical discontinuity (e.g. the surface of a tennis ball) and fiat boundary corresponds to a Human mind constructed limit (e.g. a country border). Notice that a lot of objects are indeed fiat objects; one fiat boundary is sufficient to put them in this category. For example, a stem of a champagne glass is a fiat object; it has bona fide boundaries (glass material) but a top fiat boundary (which closes the usable volume of the glass). Similarly, a "room" can also be seen as a fiat object as well when a door is open... In this paper, we will not develop the full concept of fiat objects (composed only by fiat boundaries) as it implies the description of level 2 concept of Fictional space.

The World object can be linked to Physical sub-spaces or not. Indeed, an object might exist without being linked to a sub-space (physical or even fictional). We will not detail in this paper the notion of object's identity. Linking an object to a Physical sub-space provides a dimension and an extension to the object.

A World object as a mind conceptualization can be composed of several Physical sub-spaces. It is usually implicitly associated with a given agent as explained in Level 4. Let's take the example of the "room" and let us consider a human being agent. We could consider that there is an nPPS corresponding to the walls, the roof and the floor, which is the space filled with materials and there is a PPSS filled with air. The conceptualization of the object "room" creates a separation of the PPSS into a PPSS corresponding to the air inside the "room" and a PPSS corresponding to the rest of the ambient atmosphere. These two PPSS have boundaries, some of them being in contact with nPPSS (and therefore composing the Interface) and some of them being in contact between the two PPSS. In this case, these boundaries compose an Opening between the two PPSS. The PPSS composing the object "room" is called the internal PPSS and the other one is called the external PPSS. This concept of internal and external are object dependent. Opening concept allows working out connectivity both within the object and between the inside and the outside of the object.

The Interface can also be specialized into internal interface portion and external interface portion depending on the nature of the PPSS (internal or external) involved in the touch relationships.

\section{APPLICATION TO THE 3D URBAN MODELING DOMAIN}

\subsection{Uses of Interface and Opening concepts}

The proposed ontology can be used as a basis to define a 3D urban model. Following level 5 description, the spatial extension of an object can be obtained through its links with Physical sub-spaces. There are several ways of deriving the shapes and dimensions of Physical sub-spaces, but we select a particular one involving the Interface. Indeed, most of our measuring methods actually acquire geometric information of the Interface (in other words, the geometry of some bona fide boundaries at the interface of the penetrable and non penetrable world). The exact shape is never measured; depending on the resolution and the precision we obtain a certain representation of the boundary's geometry. This implies a certain level of geometric generalization. 
Usage, Usability, and Utility of 3D City Models
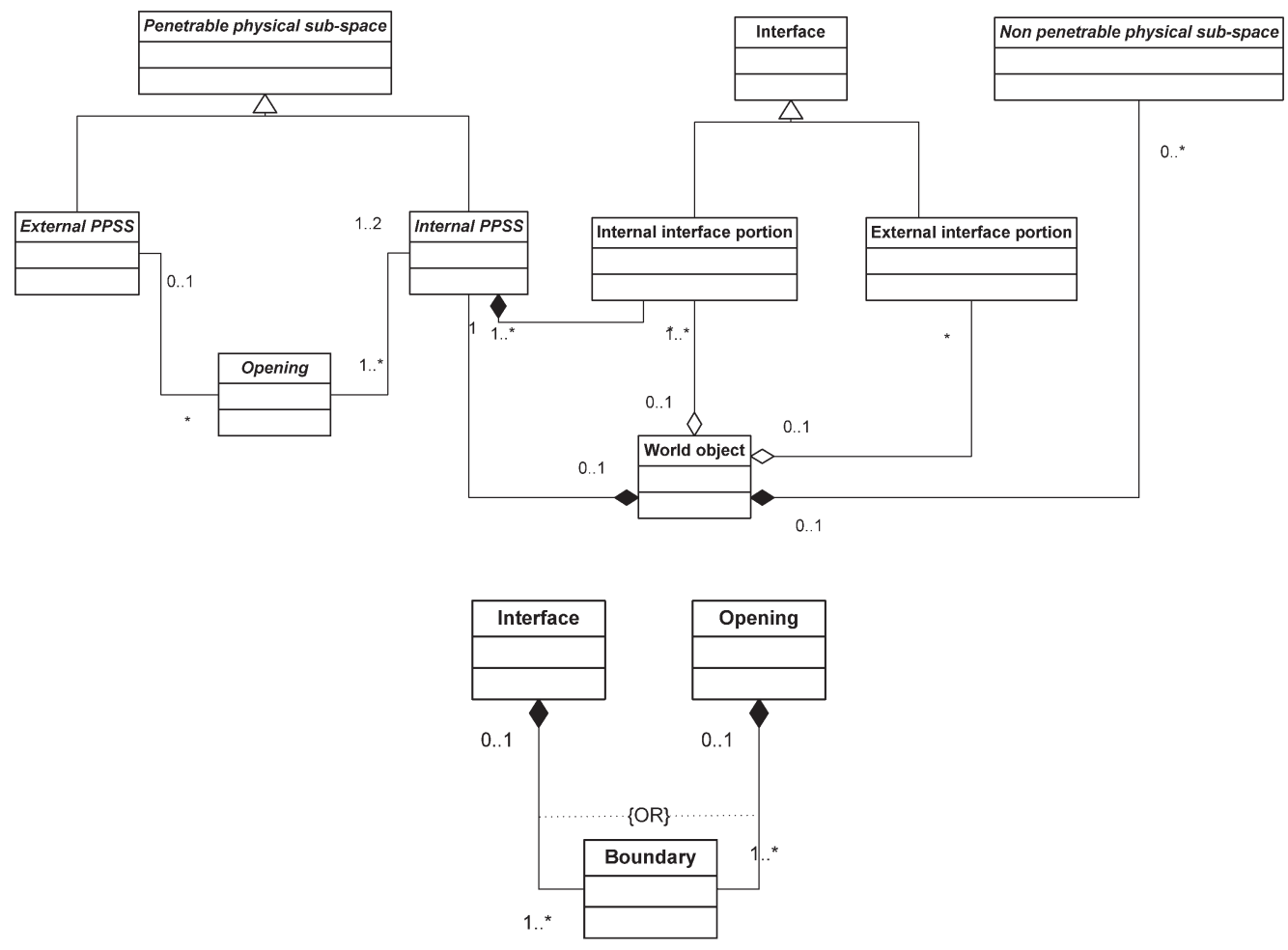

Figure 7. Proposed ontology of space - level 5.
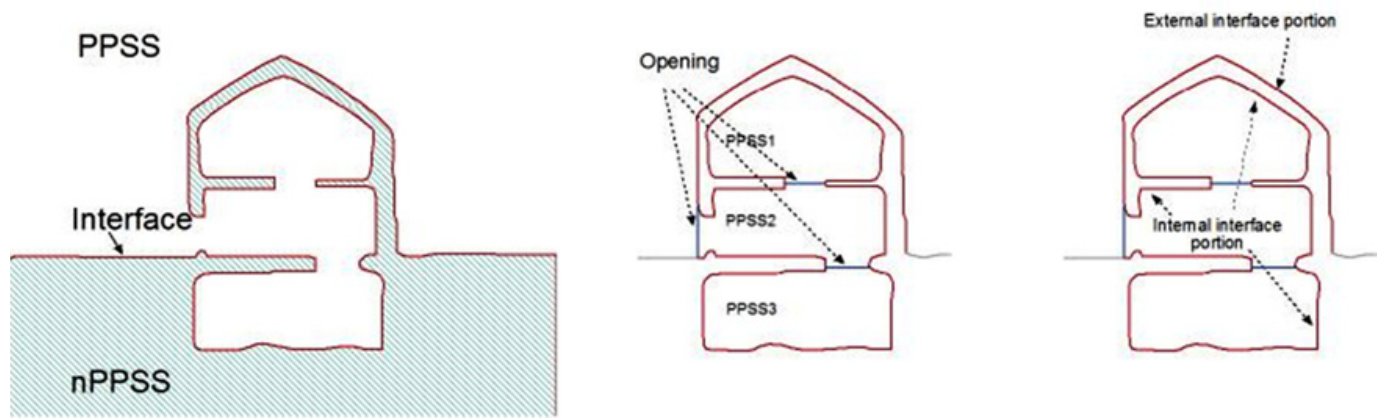

Figure 8. Ontology of space level 5 concepts applied to urban domain (traverse cut in a building).

The Figure 8 presents the concepts detailed in level 5 for a building object (represented by a traverse cut) similar to the "room" case. In this example, the nPPSS could also be subdivided in smaller pieces. This building object can be seen as composed of a several PPSS and nPPSS. These Physical sub-spaces could be generalized into higher-level physical sub-spaces, which means the building object could be linked to one generalized physical sub-space. It is indeed another type of generalization; at this stage we could use a physical space generalization or an interface geometric generalization (or both) to consider different geometric levels-of-detail. Note that this generalization could be applied also only to internal PPSS to get indoor levels-of-detail. Note that an object can be constituted only by nPPSS and only observable through a portion of the external interface ("full" object or object with inaccessible inside). 


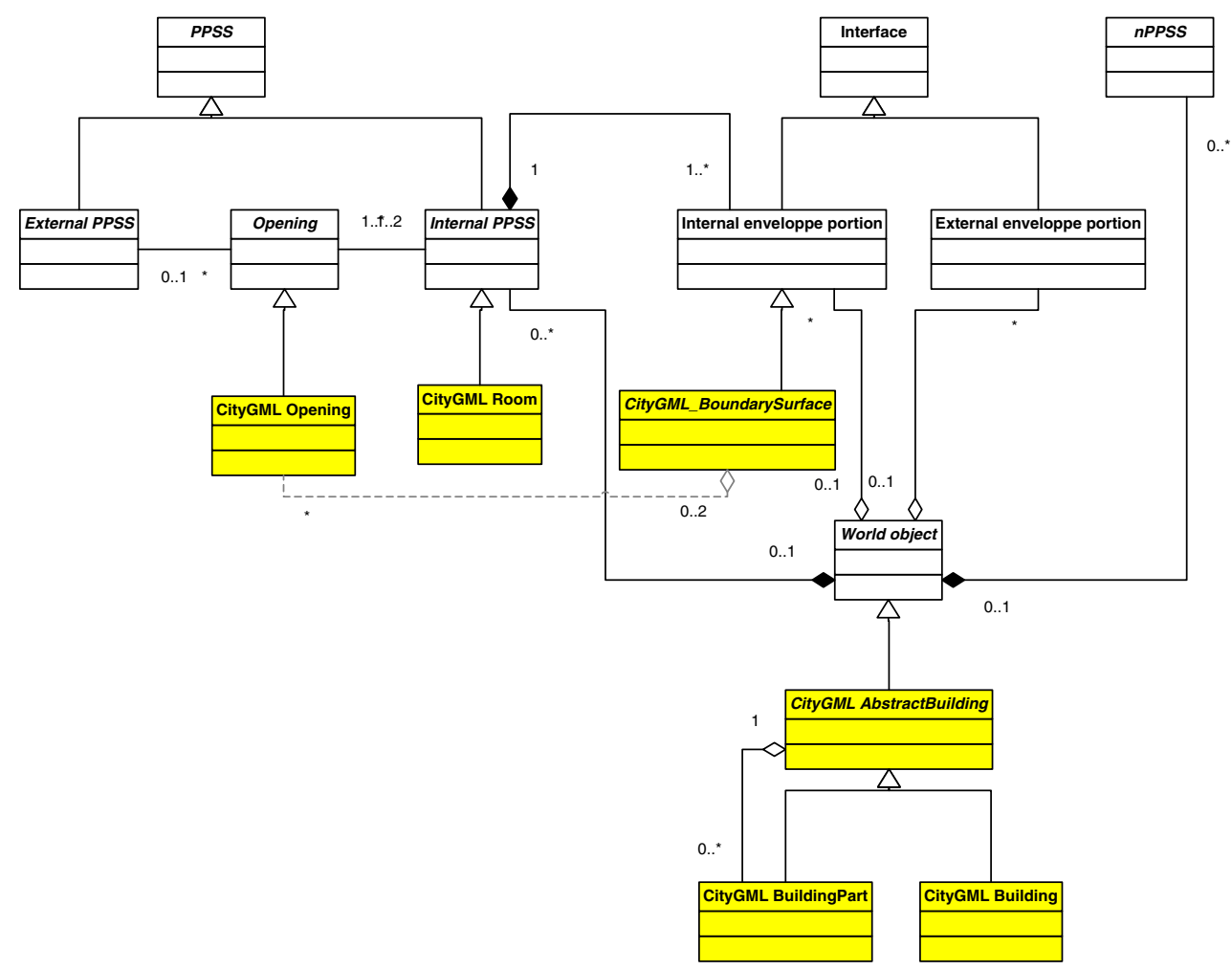

Figure 9. Application of the ontology of space (level 5) to 3D urban modeling domain and introduction of some objects of CityGML.

Similarly, it is possible to consider objects that would only be observable through their portion of internal interface (for example an underground pipe).

If the building object is itself composed of other objects (roof, walls, etc.), a semantic generalization can be added to the two cited above.

\subsection{Application to CityGML}

The defined CityGML semantic classes are composed of buildings and other man-made objects, vegetation, hydrographical objects and utilities networks. CityGML offers a pragmatic solution for modeling urban areas. As it is often the case in the world of geographical information, the ontology that CityGML designers use is implicit and therefore never clearly formalized. It appeared to us that this implicit ontology should not fundamentally differ from the one that underlies our development. The following diagram offers a reformulation according to our meta-model of a number of buildings-related objects of CityGML. Our goal is to study the correspondence between CityGML and the proposed ontology and hopefully to enrich CityGML by strengthening its ontological base.

In the case of buildings, there is a good correspondence between our ontology and CityGML (Figure 9). CityGML classes can easily be integrated in our ontology model, e.g. a CityGML "room" is a specialization of our "internal PPSS", etc. Nevertheless, some modifications can be introduced when following strictly our ontology, especially about the proposed level-of-detail. Indeed, CityGML considers five levels of details (LOD) of which four are directly linked to the representation of buildings. 


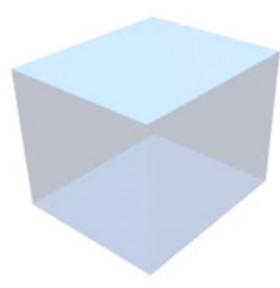

Internal LoD 1

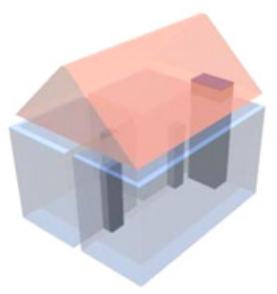

Internal LoD 2

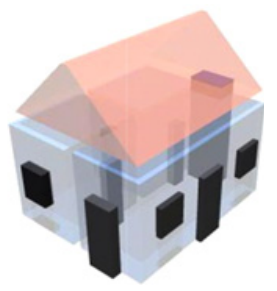

Internal LoD 3

Figure 10. Proposed concept of interior level-of-details.

- Level 1 (LOD 1): buildings are modeled as blocks with flat roofs. Models are generalized and no texture is applied.

- Level 2 (LOD 2): models of (parametric) roofs and (photorealistic or synthetic) textures are added.

- Level 3 (LOD 3): beyond an improvement in the accuracy of positioning, the difference with level 2 is in the recognition of openings.

- Level 4 (LOD 4): identical to level 3 for the outside of the building, but allows the modeling of the inside of buildings (constituent elements and openings).

First comments concern the first three level-of-details. Referring to the discussion about generalization types (section 4.1), the proposed level-of-details seem to be a mix between different types. These usagedriven LODs should beneficiate of being reformulated in terms of the proposed ontology generalization processes.

Most crucial is LOD4. Indeed, following the proposed ontology, there is no objective reason for not considering internal and interface portions geometry in the same way. We propose to expand LOD4, by considering three separate levels of detail related to a building's interior. As such, we follow our ontology's logic by considering identical levels of detail for the portions of internal interface and external interface of a building (Figure 10). The three new levels of detail that we propose are outlined below:

- Internal level of detail 1: this corresponds to one or more internal PPSS generalized by polyhedra. The internal PPSS can be generalized as a number of superimposed disconnected spaces to give an account of the notion of a floor.

- Internal level of detail 2: The internal PPSS are all represented according to some geometric generalization. The openings linking the internal sub-spaces are represented.

- Internal level of detail 3: this is identical to level 2 but without geometrical generalization and with the addition of openings on the outside. The connection between the inside of a building and the outside can therefore be envisaged only at the level of detail 3 (internal and external level of detail).

Although this proposition is still open to discussion, it shows how models could be extended by following a specific ontology.

\section{CONCLUSIONS}

In this article, we have presented a new multi-level and hierarchical approach for characterizing information, data and knowledge. The objective was to propose the basis of a simple and logical framework allowing characterizing diverse reality and knowledge representations and models. Such framework will improve common understanding of models and ontologies interrelations and dependencies. This is especially useful in Geographical Information Science that deals with huge amount of geo-information models, standards and ontologies and encounters major interoperability 
problems. This is even more critical in the 3D city modeling community which is wider and more diverse than the 2D geo-info community. The proposed multi-level and hierarchical approach is used to build a domain ontology of space, based on the assumption that a space is a n-dimensional (nD) partition of the Universe. Five hierarchical level of the ontology are defined, introducing concepts of physical space, fictional space, sub-space, boundary, interface, penetrable and non-penetrable subspace, objects, opening, internal and external sub-spaces. This ontology is then applied to the 3D urban modeling domain, with a particular focus on concepts of opening, internal and external sub-spaces. The ontology is then compared to some CityGML objects. There is a good correspondence between the applied ontology and the 3D OGC standard. However, we have identified that the way level-of-detail are used in CityGML do not fit with the applied ontology. This has leaded us to propose a concept of interior level of details. This final proposition illustrates that considering ontological foundation of data models (and standards) strengthen them by bringing justification to models enrichment or extensions, and consequently improve interoperability.

\section{References}

[1] R. L. Ackoff, From Data to Wisdom. Journal of Applies Systems Analysis, 16, 3-9 (1989)

[2] R. Studer, V.R. Benjamins, D. Fensel, Knowledge engineering: Principles and methods, Data Knowl. Eng., 25(1-2), 161-197 (1998)

[3] M. Boisot, A. Canals, Data, information and knowledge: have we got it right? Journal of Evolutionary Economics 14(1), 43-67 (2004)

[4] N. Pelekis, B. Theodoulidis, I. Kopanakis, Y. Theodoridis, Literature review of spatio-temporal database models. Knowledge Engineering Review 19(3), 235-274 (2004)

[5] M. Minout, Modélisation des Aspects Temporels dans les Bases de Données Spatiales, PhD thesis, Université Libre de Bruxelles (2007)

[6] JTS, Available on http://www.vividsolutions.com/jts/JTSHome.htm (2010)

[7] C. Zaki, E. Zekri, M. Servières, G. Moreau, G. Hégron, Urban spatiotemporal data-modeling: application to the study of pedestrian walkways. Advances in Intelligent Decision Technologies, R.J. Howlett, L.C. Jain, G. Phillips-Wren, and K. Nakamatsu, eds., Baltimore, MD, USA, 2010, 549-558 (2010)

[8] C. Parent, S. Spaccapietra, E. Zimányi, Modeling /Spatial Data in the MADS Conceptual Model MADS, Spatial Data Handling '98 Conference Proceedings, Vancouver, BC, Canada: 138-150 (1998)

[9] Y. Bedard, Visual Modeling of Spatial Databases Towards Spatial Extensions and UML, Geomatica, 53(2), 169-186 (1999)

[10] M. Uschold, T. King, Towards a methodology for building ontologies, in Proceedings IJCAI-95, Workshop on Basic Ontological Issues in Knowledge Sharing, Canada (1995)

[11] G. vanHeijst, A. Schreiber, B. Wielinga, Using explicit ontologies in kbs development, Int. J. of Human-Computer Studies, 46(2/3), 183-292 (1997)

[12] G. Diallo, Une Architecture à Base d'Ontologies pour la Gestion Unifiée des Données Structurées et non Structurées, PhD thesis, Université Joseph Fourier - Grenoble I (2006)

[13] B. Chandrasekaran, J. Josephson, V.R. Benjamins, The ontology of tasks and methods, Knowledge Acquisition Workshop, KAW98 (1998)

[14] Open Geospatial Consortium Inc, OpenGIS®City Geography Markup Language (CityGML) Encoding Standard, OGC 08-007r1, http://www.opengeospatial.org/standards/ citygml (2008)

[15] M. Fowler, K. Scott, UML Distilled - Applying the Standard Object Modeling Language, Addison-Wesley (1998) 
Usage, Usability, and Utility of 3D City Models

[16] R. Billen, F. Laplanche, S., Zlatanova, S. Emgard, Vers la création d'un méta-modèle générique de l'information spatiale 3D urbaine, 37-42 (XYZ) (2008)

[17] R. Billen, Exploring the needs and applicability of a 3D urban land register information system, London, UK: Royal Institution of Chartered Surveyors, RICS (2009)

[18] B. Smith, A. Varzi, Fiat and Bona Fide Boundaries, Philosophy and Phenomenological Research 60(2), 401-420 (2000) 